Quantum Field Theory Under the Influence

of External Conditions (QFEXT11)

International Journal of Modern Physics: Conference Series

Vol. 14 (2012) 181-199

(C) World Scientific Publishing Company

DOI: $10.1142 / \mathrm{S} 2010194512007325$

\title{
REPULSIVE CASIMIR EFFECTS
}

\author{
K. A. MILTON, E. K. ABALO, PRACHI PARASHAR, NIMA POURTOLAMI \\ Homer L. Dodge Department of Physics and Astronomy, University of Oklahoma, \\ Norman, OK 73019 USA \\ milton@nhn.ou.edu,abalo@nhn.ou.edu,prachi@nhn.ou.edu,nimap@ou.edu \\ IVER BREVIK and S. Å. ELLINGSEN \\ Department of Energy and Process Engineering, Norwegian University of Science and \\ Technology, N-7491 Trondheim, Norway \\ iver.h.brevik@ntnu.no, simen.a.ellingsen@ntnu.no
}

Published 28 July 2012

\begin{abstract}
Like Casimir's original force between conducting plates in vacuum, Casimir forces are usually attractive. But repulsive Casimir forces can be achieved in special circumstances. These might prove useful in nanotechnology. We give examples of when repulsive quantum vacuum forces can arise with conducting materials.
\end{abstract}

Keywords: Casimir forces, Casimir repulsion, Casimir-Polder interactions

PACS numbers: 42.50.Lc, 32.10.Dk, 12.20.-m, 03.50.De

\section{Multiple Scattering Technique}

The multiple scattering approach starts from the well-known formula for the electromagnetic quantum vacuum energy or Casimir energy ( $\tau$ is the "infinite" time that the configuration exists $)^{1}$

$$
E=\frac{i}{2 \tau} \operatorname{Tr} \ln \boldsymbol{\Gamma} \rightarrow \frac{i}{2 \tau} \operatorname{Tr} \ln \boldsymbol{\Gamma} \boldsymbol{\Gamma}_{0}^{-1}
$$

where $\boldsymbol{\Gamma}$ is the Green's dyadic satisfying

$$
\left(\frac{1}{\omega^{2}} \nabla \times \frac{1}{\mu} \nabla-\varepsilon\right) \Gamma=\mathbf{1}
$$

while $\boldsymbol{\Gamma}_{0}$ satisfies the same equation with $\varepsilon=\mu=1$ everywhere. We will choose the Green's dyadics satisfying outgoing-wave boundary conditions (corresponding to the Feynman propagator), although other choices can be made. 
Consider material bodies characterized by a permittivity $\varepsilon(\mathbf{r})$ and a permeability $\mu(\mathbf{r})$, so we have corresponding electric and magnetic potentials

$$
V_{e}(\mathbf{r})=\varepsilon(\mathbf{r})-1, \quad \text { and } \quad V_{m}(\mathbf{r})=\mu(\mathbf{r})-1 .
$$

Then the trace-log appearing in the vacuum energy is $\left(\boldsymbol{\Phi}_{0}=-\frac{1}{\zeta} \boldsymbol{\nabla} \times \boldsymbol{\Gamma}_{0}\right)$

$$
\begin{aligned}
\operatorname{Tr} \ln \boldsymbol{\Gamma} \boldsymbol{\Gamma}_{0}^{-1}= & -\operatorname{Tr} \ln \left(\mathbf{1}-\boldsymbol{\Gamma}_{0} V_{e}\right)-\operatorname{Tr} \ln \left(\mathbf{1}-\boldsymbol{\Gamma}_{0} V_{m}\right) \\
& -\operatorname{Tr} \ln \left(\mathbf{1}+\boldsymbol{\Phi}_{0} \mathbf{T}_{e} \boldsymbol{\Phi}_{0} \mathbf{T}_{m}\right),
\end{aligned}
$$

in terms of the $\mathbf{T}$-matrix,

$$
\mathbf{T}_{e, m}=V_{e, m}\left(\mathbf{1}-\boldsymbol{\Gamma}_{0} V_{e, m}\right)^{-1} .
$$

If we have disjoint electric bodies, the interaction term separates out:

$$
\begin{aligned}
\operatorname{Tr} \ln \left(\mathbf{1}-\boldsymbol{\Gamma}_{0}\left(V_{1}+V_{2}\right)\right) & =-\operatorname{Tr} \ln \left(\mathbf{1}-\boldsymbol{\Gamma}_{0} \mathbf{T}_{1}\right) \\
-\operatorname{Tr} \ln \left(\mathbf{1}-\boldsymbol{\Gamma}_{0} \mathbf{T}_{2}\right) & -\operatorname{Tr} \ln \left(\mathbf{1}-\boldsymbol{\Gamma}_{0} \mathbf{T}_{1} \boldsymbol{\Gamma}_{0} \mathbf{T}_{2}\right),
\end{aligned}
$$

so only the latter term contributes to the interaction energy,

$$
E_{\mathrm{int}}=\frac{i}{2} \operatorname{Tr} \ln \left(\mathbf{1}-\boldsymbol{\Gamma}_{0} \mathbf{T}_{1} \boldsymbol{\Gamma}_{0} \mathbf{T}_{2}\right)
$$

The same is true if one body is electric and the other magnetic,

$$
E_{\text {int }}=-\frac{i}{2} \operatorname{Tr} \ln \left(1+\mathbf{\Phi}_{0} \mathbf{T}_{1}^{e} \boldsymbol{\Phi}_{0} \mathbf{T}_{2}^{m}\right) .
$$

Using this, it is straightforward to show that the Lifshitz energy per area between parallel dielectric and diamagnetic slabs, separated by a distance $a$, is

$$
\mathcal{E}_{\varepsilon \mu}=\frac{1}{16 \pi^{3}} \int d \zeta \int d^{2} k\left[\ln \left(1-r_{1} r_{2}^{\prime} e^{-2 \kappa a}\right)+\ln \left(1-r_{1}^{\prime} r_{2} e^{-2 \kappa a}\right)\right],
$$

where

$$
r_{i}=\frac{\kappa-\kappa_{i}}{\kappa+\kappa_{i}}, \quad r_{i}^{\prime}=\frac{\kappa-\kappa_{i}^{\prime}}{\kappa+\kappa_{i}^{\prime}}
$$

with

$$
\kappa^{2}=k^{2}+\zeta^{2}, \quad \kappa_{1}^{2}=k^{2}+\varepsilon \zeta^{2}, \quad \kappa_{1}^{\prime}=\kappa_{1} / \varepsilon, \quad \kappa_{2}^{2}=k^{2}+\mu \zeta^{2}, \quad \kappa_{2}^{\prime}=\kappa_{2} / \mu .
$$

This means in the perfect reflecting limit, $\varepsilon \rightarrow \infty, \mu \rightarrow \infty$,

$$
\mathcal{E}_{\text {Boyer }}=+\frac{7}{8} \frac{\pi^{2}}{720 a^{3}},
$$

we get Boyer's repulsive result. ${ }^{3}$

It is also well known, apparent from the purely electric version of (9), in the Lifshitz-Dzyaloshinskii-Pitaevskii situation ${ }^{4}$ of parallel dielectric media, with the intermediate medium having an intermediate value of the permittivity:

$$
\varepsilon_{1}>\varepsilon_{3}>\varepsilon_{2}
$$

there is a Casimir repulsion between the upper and lower media. This was demonstrated in the Munday-Capasso-Persegian experiment. ${ }^{5}$ 


\section{Casimir Effect on Spheres and Cylinders}

Earlier Boyer had shown ${ }^{6}$ that the Casimir self-energy of a spherical shell was positive, that is, repulsive. Such calculations have been generalized, as displayed in Table 1. Note that all these energies for spheres are positive (repulsive). Very recently, energies for cylinders with Dirichlet boundaries having triangular cross sections were computed, which also display positive Casimir self-energies, ${ }^{16}$ as we discuss in Sec. 4 below.

\section{Dimensional Dependence}

Bender and Milton ${ }^{8}$ considered the Casimir effect due to fluctuations in a scalar field interior and exterior to a Dirichlet hypersphere, in $D$ spatial dimensions, and found that poles occur in even spatial dimensions, as shown in Fig. 1, which shows that for a scalar field, subject to Dirichlet boundary conditions on the hyperspherical surface, repulsion occurs for $2<D<4$.

\section{Triangular Cylinders}

For an equilateral triangle of height $h$, the scalar eigenmodes corresponding to Dirichlet boundary conditions are known explicitly ${ }^{17,18}$

$$
\gamma_{l}^{2}=\frac{2}{3}\left(\frac{\pi}{h}\right)^{2}\left(l_{1}^{2}+l_{2}^{2}+l_{3}^{2}\right), \quad l_{1}+l_{2}+l_{3}=0, \quad l_{i} \neq 0
$$

Although this has been appreciated for most of a century, only last year was the corresponding calculation of the Casimir energy for a cylinder with such a triangular cross section (and also those obtained by bisecting an equilateral triangle and a square) carried out. ${ }^{16}$

Table 1. Casimir energy $(E)$ for a sphere and Casimir energy per unit length $(\mathcal{E})$ for an infinite cylinder, both of radius $a$. Here the different boundary conditions are perfectly conducting for electromagnetic fields (EM), Dirichlet for scalar fields (D), dilute dielectric for electromagnetic fields [coefficient of $(\varepsilon-1)^{2}$ ], dilute dielectric for electromagnetic fields with media having the same speed of light (coefficient of $\xi^{2}=[(\varepsilon-1) /(\varepsilon+1)]^{2}$ ), and weak coupling for scalar field with $\delta$-function boundary (coefficient of $\lambda^{2} / a^{2}$ ). The references given are, to the authors' knowledge, the first paper in which the results in the various cases were found.

\begin{tabular}{lll}
\hline Type & $E_{\text {Sphere }} a$ & $\mathcal{E}_{\text {Cylinder }} a^{2}$ \\
\hline EM & $+0.04618^{6}$ & $-0.01356^{7}$ \\
$\mathrm{D}$ & $+0.002817^{8}$ & $+0.0006148^{9}$ \\
$(\varepsilon-1)^{2}$ & $+0.004767=\frac{23}{1536 \pi} 10$ & $0^{11}$ \\
$\xi^{2}$ & $+0.04974=\frac{5}{32 \pi} 12$ & $0^{13}$ \\
$\lambda^{2} / a^{2}$ & $+0.009947=\frac{1}{32 \pi} 14$ & $0^{15}$ \\
\hline
\end{tabular}




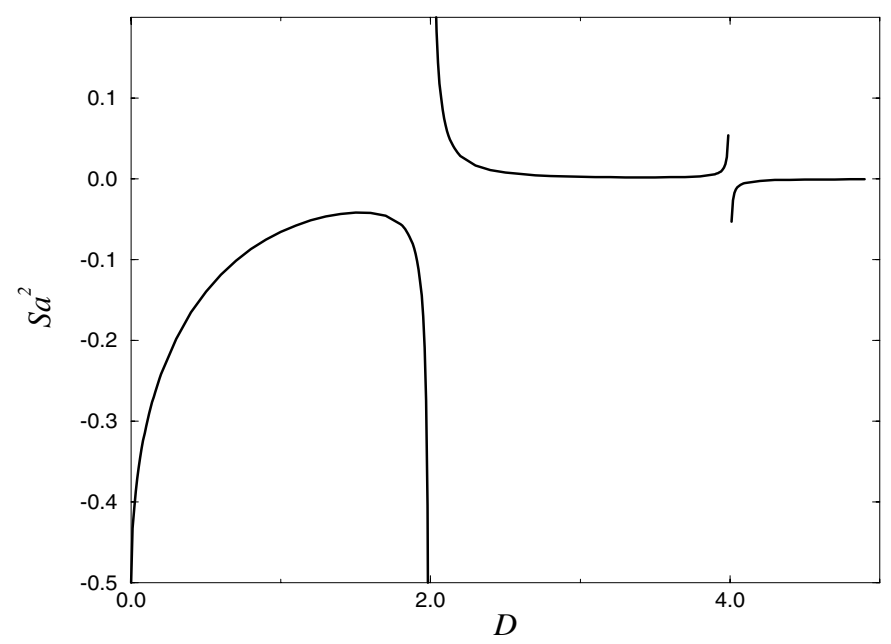

Figure 1. Scalar Casimir stress $\mathcal{S}$ for $0<D<5$ on a spherical shell. imposing Dirichlet boundary conditions. Here $D$ is the number of spatial dimensions.

In $d$ longitudinal dimensions, the Casimir energy is

$$
\mathcal{E}=-\frac{\Gamma(-1 / 2-d / 2)}{2^{2+d} \pi^{(d+1) / 2}} \sum_{l}\left(\gamma_{l}^{2}\right)^{(d+1) / 2},
$$

which can be analytically continued and summed by means of the exceedingly rapidly convergent Chowla-Selberg formula, ${ }^{19}$

$$
\mathcal{E}=+\frac{0.0177891}{h^{2}}
$$

We can also evaluate the eigenvalue sum by use of the Poisson sum formula,

$$
\sum_{l=-\infty}^{\infty} f(l)=2 \pi \sum_{k=-\infty}^{\infty} \tilde{f}(k),
$$

in terms of the Fourier transform

$$
\tilde{f}(k)=\int_{-\infty}^{\infty} \frac{d \alpha}{2 \pi} e^{2 \pi i k \alpha} f(\alpha) .
$$

We use the Poisson sum formula together with (temporal) point-splitting regularization, starting from

$$
E=\frac{1}{2 i} \int(d \mathbf{r}) \int \frac{d \omega}{2 \pi} 2 \omega^{2} \mathcal{G}(\mathbf{r}, \mathbf{r}) e^{-i \omega \tau}
$$


with $\tau \rightarrow 0$, which for a cylindrical waveguide gives for the energy per unit length

$$
\begin{aligned}
\mathcal{E} & =\frac{1}{2} \int_{-\infty}^{\infty} \frac{d \zeta}{2 \pi} 2\left(-\zeta^{2}\right) \int \frac{d k}{2 \pi} \sum_{m, n} \frac{1}{\zeta^{2}+k^{2}+\gamma_{m n}^{2}} e^{i \zeta \tau} \\
& =\frac{1}{2} \lim _{\tau \rightarrow 0}\left(-\frac{d}{d \tau}\right) \int_{-\infty}^{\infty} \frac{d k}{2 \pi} \sum_{m, n} e^{-\tau \sqrt{k^{2}+\gamma_{m n}^{2}}}
\end{aligned}
$$

A virtue of the point-splitting method is that we can isolate the divergences in the energy:

$$
\widehat{\mathcal{E}}_{\mathrm{Eq}}^{(D)}=\lim _{\tau \rightarrow 0}\left(\frac{3 A}{2 \pi^{2} \tau^{4}}-\frac{P}{8 \pi \tau^{3}}+\frac{1}{6 \pi \tau^{2}}\right) .
$$

We note that the "volume" and "surface" divergent terms, which are respectively proportional to the area of the triangle $A=h^{2} / \sqrt{3}$ and the perimeter $P=2 \sqrt{3} h$, are as expected, and are presumably not of physical relevance. The last term, a constant in $h$, certainly does not contribute to the self-stress on the cylinder. Only this term reflects the corner divergences. For a general polygon, with interior angles $\alpha_{i}$, the last term is

$$
\frac{1}{48 \pi} \sum_{i}\left(\frac{\pi}{\alpha_{i}}-\frac{\alpha_{i}}{\pi}\right) \frac{1}{\tau^{2}}
$$

These coefficients are proportional to the heat kernel coefficients - in particular there is no $a_{2}$ heat kernel coefficient, because the surfaces are flat, which means that the Casimir energy can be identified unambiguously.

Remarkably, for the integrable polygonal figures we are considering, the Casimir energy can be given in closed form, in terms of the polygamma function. Thus

$$
\mathcal{E}_{\mathrm{Eq}}^{(D)}=-\frac{1}{96 h^{2}}\left[\frac{\sqrt{3}}{9}\left[\psi^{\prime}(1 / 3)-\psi^{\prime}(2 / 3)\right]-\frac{8}{\pi} \zeta(3)\right]=\frac{0.0177891}{h^{2}}
$$

It is a priori remarkable that such an explicit form can be achieved for a strongcoupling problem.

The same methods can be used to evaluate the Casimir energy for a square waveguide (side $a$ ), a well-studied system, ${ }^{20,21}$ although the closed form was previously unknown,

$$
\begin{aligned}
\mathcal{E}_{\mathrm{Sq}}^{(D)} & =-\frac{1}{32 \pi^{2} a^{2}}\left[2 \zeta(4)-\pi \zeta(3)+8 \pi^{2} \sum_{l=1}^{\infty} l^{3 / 2} \sigma_{3}(l) K_{3 / 2}(2 \pi l)\right] \\
& =-\frac{1}{32 \pi^{2} a^{2}}\left[4 \zeta(4)-2 \pi \zeta(3)+4 \sum_{k, l=1}^{\infty} \frac{1}{\left(k^{2}+l^{2}\right)^{2}}\right] \\
& =\frac{1}{16 \pi a^{2}}\left[\zeta(3)-\frac{\pi}{3} G\right]=\frac{0.00483155}{a^{2}} .
\end{aligned}
$$




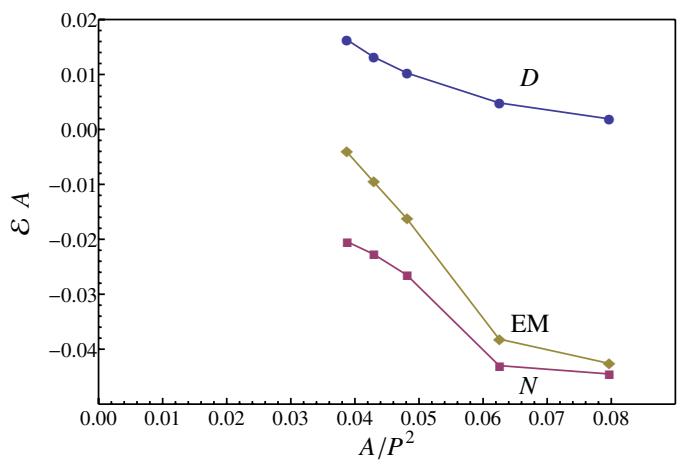

Figure 2. Dependence of Casimir energies of triangular waveguides. The top curve is for Dirichlet boundary conditions, the bottom for Neumann boundary conditions, and the intermediate curve represents surfaces which are perfect electromagnetic conductors.

By bifurcating the square, we can obtain the isosceles right triangle, and by bifurcating the equilateral triangle we can get the $30^{\circ}-60^{\circ}-90^{\circ}$ triangle:

$$
\begin{aligned}
& \mathcal{E}_{\mathrm{Iso}}^{(D)}=\frac{1}{2} \mathcal{E}_{\mathrm{Sq}}^{D)}+\frac{\zeta(3)}{16 \pi a^{2}}=\frac{0.0263299}{a^{2}}, \\
& \mathcal{E}_{369}^{(D)}=\frac{1}{2} \mathcal{E}_{\mathrm{Eq}}^{(D)}+\frac{\zeta(3)}{8 \pi h^{2}}=\frac{0.0567229}{h^{2}},
\end{aligned}
$$

to be compared to the result for a circle ${ }^{9}$

$$
\mathcal{E}_{\text {Circ }}^{(D)}=\frac{0.0006148}{a^{2}}
$$

For the latter, external contributions must be included, to cancel the curvature divergences.

We can also get results for Neumann boundary conditions ( $\mathrm{H}$ or TE modes)

$$
\begin{aligned}
& \mathcal{E}_{\mathrm{Sq}}^{(N)}=\mathcal{E}_{\mathrm{Sq}}^{(D)}-\frac{\zeta(3)}{8 \pi a^{2}}=-\frac{0.0429968}{a^{2}}, \\
& \mathcal{E}_{\mathrm{Eq}}^{(N)}=\mathcal{E}_{\mathrm{Eq}}^{(D)}-\frac{\zeta(3)}{6 \pi h^{2}}=-\frac{0.045982}{h^{2}}, \\
& \mathcal{E}_{\mathrm{Iso}}^{(N)}=\frac{1}{2} \mathcal{E}_{\mathrm{Sq}}^{(N)}-\frac{\zeta(3)}{16 \pi a^{2}}=-\frac{0.0454125}{a^{2}}, \\
& \mathcal{E}_{369}^{(N)}=\frac{1}{2} \mathcal{E}_{\mathrm{Eq}}^{(N)}-\frac{\zeta(3)}{8 \pi h^{2}}=-\frac{0.0708193}{h^{2}} .
\end{aligned}
$$

Graph 2 shows the systematic dependence of $\mathcal{E}^{(D)}, \mathcal{E}^{(N)}$ and $\mathcal{E}^{(\mathrm{EM})}$ expressed in the dimensionless form $\mathcal{E} A$ in terms of the geometrical quantity $\left(A / P^{2}\right)$, where $A$ is the cross-sectional area, and $P$ is the cross-sectional perimeter of the waveguide. The limited analytic results have been supplemented by a numerical method to extract eigenvalues for right triangles with arbitrary acute angles. Those results, 


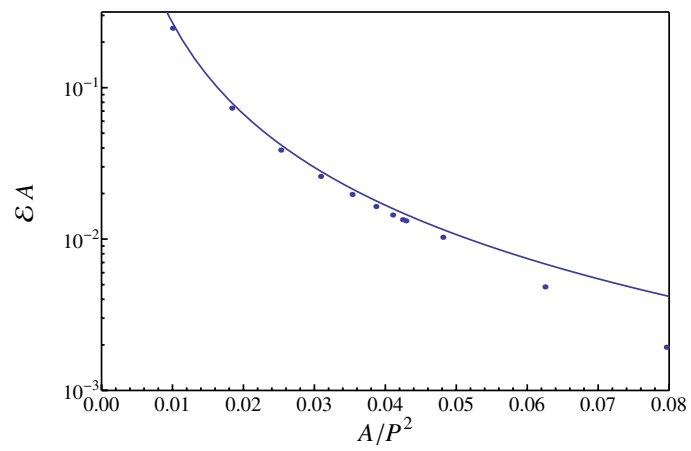

Figure 3. Scaled Dirichlet Casimir energies for triangular waveguides both for numerical and analytically solvable cases. The two rightmost points correspond to a square and a circular cross section. The solid line is the PFA approximation.

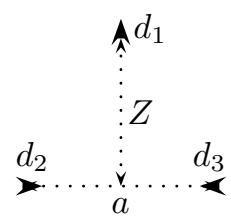

Figure 4. Configuration of three dipoles, two of which are antiparallel, and one perpendicular to the other two.

shown in Fig. 3 for the Dirichlet case, lie on our universal curve, and agree with the proximity force approximation PFA (solid line) for small acute angles:

$$
\mathcal{E}_{\mathrm{PFA}}^{(D)} A=-\frac{\pi^{2}}{1440} \int_{0}^{a} \frac{d r}{(r \theta)^{3}} \frac{1}{2} a^{2} \theta \rightarrow \frac{\pi^{2}}{368640}\left(\frac{P^{2}}{A}\right)^{2} .
$$

\section{Classical Repulsion}

Both classical and quantum repulsion were described last year by Levin et al. ${ }^{22}$, and we give some additional examples here. (More details of our considerations appear in Ref. 23.)

\subsection{Classical dipole interaction}

It is possible to achieve a repulsive force between a configuration of fixed dipoles. Consider the situation illustrated in Fig. 4. Here we have two dipoles, of strength $d_{2}$ and $d_{3}$ lying along the $x$ axis, separated by a distance $a$. A third dipole of strength 


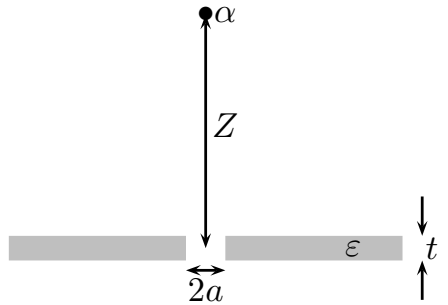

Figure 5. Three-dimensional geometry of a dipole or polarizable atom a distance $Z$ above a dielectric or conducting plate with a circular aperture of radius $a$. In this paper we will consider a conductor with thickness $t=0$.

$d_{1}$ lies along the $z$ axis. If the two parallel dipoles are oppositely directed and of equal strength,

$$
\mathbf{d}_{2}=-\mathbf{d}_{3}=d_{2} \hat{\mathbf{x}}
$$

equally distant from the $z$ axis, and the dipole on the $z$ axis is directed along that axis,

$$
\mathbf{d}_{1}=d_{1} \hat{\mathbf{z}}
$$

the force on that dipole is along the $z$ axis:

$$
F_{z}=3 a d_{1} d_{2} \frac{a^{2} / 4-4 Z^{2}}{\left(Z^{2}+a^{2} / 4\right)^{7 / 2}}
$$

which changes $\operatorname{sign}$ at $Z=a / 4$; that is, close to $Z=0$ the force on dipole 1 is repulsive.

\subsection{Interaction of atom with aperture}

We first consider a dipole above an aperture in a perfectly conducting line in two dimensions, as shown in Fig. 5. The Green's function which vanishes on the entire line $z=0$ is

$$
G\left(\mathbf{r}, \mathbf{r}^{\prime}\right)=-\ln \left[\left(x-x^{\prime}\right)^{2}+\left(z-z^{\prime}\right)^{2}\right]+\ln \left[\left(x-x^{\prime}\right)^{2}+\left(z+z^{\prime}\right)^{2}\right],
$$

with the boundary condition:

$$
G\left(x, 0 ; x^{\prime}, z^{\prime}\right)=0 .
$$

Then the electrostatic potential at any point above the $z=0$ line is

$$
\phi(\mathbf{r})=\int_{z>0}\left(d \mathbf{r}^{\prime}\right) G\left(\mathbf{r}, \mathbf{r}^{\prime}\right) \rho\left(\mathbf{r}^{\prime}\right)+\frac{1}{4 \pi} \int_{\text {ap }} d S^{\prime} \frac{\partial}{\partial z^{\prime}} G\left(\mathbf{r}, \mathbf{r}^{\prime}\right) \phi\left(\mathbf{r}^{\prime}\right),
$$

where

$$
\rho(\mathbf{r})=-\mathbf{d} \cdot \boldsymbol{\nabla} \delta(\mathbf{r}-\mathbf{R}), \quad \mathbf{R}=(0, Z) .
$$

The surface integral extends only over the aperture because the potential vanishes on the conducting line. If we choose $\mathbf{d}$ to point along the $z$ axis we easily find $(2 a=$ 
width of aperture)

$$
\begin{aligned}
\phi(x, z>0)= & 2 d\left[\frac{z-Z}{x^{2}+(z-Z)^{2}}+\frac{z+Z}{x^{2}+(z+Z)^{2}}\right] \\
& +\frac{1}{\pi} \int_{-a}^{a} d x^{\prime} \frac{z}{\left(x-x^{\prime}\right)^{2}+z^{2}} \phi\left(x^{\prime}, 0\right) .
\end{aligned}
$$

Now the free Green's function in two dimensions is

$$
G_{0}\left(\mathbf{r}, \mathbf{r}^{\prime}\right)=4 \pi \int \frac{(d \mathbf{k})}{(2 \pi)^{2}} \frac{e^{i k_{x}\left(x-x^{\prime}\right)} e^{i k_{z}\left(z-z^{\prime}\right)}}{k_{x}^{2}+k_{z}^{2}}=\int_{-\infty}^{\infty} d k_{x} \frac{1}{\left|k_{x}\right|} e^{i k_{x}\left(x-x^{\prime}\right)} e^{-\left|k_{x}\right|\left|z-z^{\prime}\right|} \text {. }
$$

Then the surface integral above is

$$
\int_{-\infty}^{\infty} \frac{d k_{x}}{2 \pi} e^{i k_{x} x} e^{-\left|k_{x}\right| z} \tilde{\phi}\left(k_{x}\right),
$$

in terms of the Fourier transform of the field

$$
\tilde{\phi}\left(k_{x}\right)=\int_{-\infty}^{\infty} d x^{\prime} e^{-i k_{x} x^{\prime}} \phi\left(x^{\prime}, 0\right)=2 \int_{0}^{a} d x^{\prime} \cos k_{x} x^{\prime} \phi\left(x^{\prime}, 0\right),
$$

since $\phi(x, 0)$ must be an even function for the geometry considered. Thus we conclude

$$
\phi(x, z>0)=2 d\left[\frac{z-Z}{x^{2}+(z-Z)^{2}}+\frac{z+Z}{x^{2}+(z+Z)^{2}}\right]+\frac{1}{\pi} \int_{0}^{\infty} d k \cos k x e^{-k z} \tilde{\phi}(k) .
$$

The electric field in the aperture is

$$
E_{z}(x, z=0+)=-\left.\frac{\partial}{\partial z} \phi(x, z)\right|_{z=0+}=-4 d \frac{x^{2}-Z^{2}}{\left(x^{2}+Z^{2}\right)^{2}}+\frac{1}{\pi} \int_{0}^{\infty} d k k \cos k x \tilde{\phi}(k) .
$$

On the other side of the aperture, there is no charge density, so for $z<0$ the potential is

$$
\phi(x, z<0)=\frac{1}{\pi} \int_{0}^{\infty} d k \cos k x e^{k z} \tilde{\phi}(k),
$$

so the $z$-component of the electric field in the aperture is

$$
E_{z}(x, z=0-)=-\left.\frac{\partial}{\partial z} \phi(x, z)\right|_{z=0-}=-\frac{1}{\pi} \int_{0}^{\infty} d k k \cos k x \tilde{\phi}(k) .
$$

Because we require that the electric field be continuous in the aperture, and the potential vanish on the conductor, we obtain the two coupled integral equations for this problem,

$$
\begin{aligned}
4 d \frac{x^{2}-Z^{2}}{\left(x^{2}+Z^{2}\right)^{2}} & =\frac{2}{\pi} \int_{0}^{\infty} d k k \cos k x \tilde{\phi}(k), \quad 0<|x|<a, \\
0 & =\int_{0}^{\infty} d k \cos k x \tilde{\phi}(k), \quad|x|>a .
\end{aligned}
$$


In fact, these equations have a simple solution ${ }^{24}$

$$
\tilde{\phi}(k)=-\frac{2 Z d \pi}{a} \int_{0}^{1} d x x \frac{J_{0}(k a x)}{\left(x^{2}+Z^{2} / a^{2}\right)^{3 / 2}} .
$$

From this, we can work out the energy of the system from

$$
U=-\frac{1}{2} d E_{z}(0, Z)=\left.\frac{1}{2} d \frac{\partial \phi}{\partial z}\right|_{z=Z, x=0},
$$

where the factor of $1 / 2$ comes from the fact that this must be the energy required to assemble the system. We must further drop the self-energy of the dipole due to its own field. We are then left with

$$
\begin{aligned}
U_{\text {int }} & =-\frac{d^{2}}{4 Z^{2}}-\frac{d}{2 \pi} \int_{0}^{\infty} d k k e^{-k Z \tilde{\phi}(k)} \\
& =-\frac{d^{2}}{4 Z^{2}}+\frac{Z^{2} d^{2}}{a^{4}} \int_{0}^{1} \frac{1}{2} d x^{2} \frac{1}{\left(x^{2}+Z^{2} / a^{2}\right)^{3}} \\
& =-\frac{1}{4} \frac{Z^{2} d^{2}}{\left(a^{2}+Z^{2}\right)^{2}},
\end{aligned}
$$

twice that of Levin et al. ${ }^{22}$ Since this vanishes at $Z=0$ and $Z=\infty$, the force must change from attractive to repulsive, which happens at $Z=a$.

\subsection{Circular aperture interacting with dipole}

It is quite straightforward to repeat the above calculation in three dimensions. Again we are considering a dipole, polarized on the symmetry axis, a distance $Z$ above a circular aperture of radius $a$ in a conducting plate, illustrated in Fig. 5.

The free three-dimensional Green's function in cylindrical coordinates has the representation

$$
\frac{1}{\sqrt{\rho^{2}+z^{2}}}=\int_{0}^{\infty} d k J_{0}(k \rho) e^{-k|z|},
$$

and so if we follow the above procedure we find for the potential above the plate

$$
\begin{aligned}
\phi\left(\mathbf{r}_{\perp}, z>0\right)=d & {\left[\frac{z-Z}{\left[r_{\perp}^{2}+(z-Z)^{2}\right]^{3 / 2}}+\frac{z+Z}{\left[r_{\perp}^{2}+(z+Z)^{2}\right]^{3 / 2}}\right] } \\
& +\int_{0}^{\infty} d k k e^{-k z} J_{0}\left(k r_{\perp}\right) \Phi(k)
\end{aligned}
$$

where the Bessel transform of the potential in the aperture is

$$
\Phi(k)=\int_{0}^{\infty} d \rho \rho J_{0}(k \rho) \phi(\rho, 0) .
$$

Thus the integral equations resulting from the continuity of the $z$-component of the electric field in the aperture and the vanishing of the potential on the conductor 
are

$$
\begin{aligned}
& d \frac{r_{\perp}^{2}-2 Z^{2}}{\left[r_{\perp}^{2}+Z^{2}\right]^{5 / 2}}=\int_{0}^{\infty} d k k^{2} J_{0}\left(k r_{\perp}\right) \Phi(k), \quad r_{\perp}<a, \\
& 0=\int_{0}^{\infty} d k k J_{0}\left(k r_{\perp}\right) \Phi(k), \quad r_{\perp}>a .
\end{aligned}
$$

The solution to these equations is given in Titchmarsh's book, ${ }^{25}$ and after a bit of manipulation we obtain

$$
\Phi(k)=-\left(\frac{2 k a}{\pi}\right)^{1 / 2} \frac{2 d Z}{k a^{2}} \int_{0}^{1} d x x^{3 / 2} \frac{J_{1 / 2}(x k a)}{\left(x^{2}+Z^{2} / a^{2}\right)^{2}} .
$$

Then the energy may be easily evaluated using

$$
\int_{0}^{\infty} d k k^{3 / 2} e^{-k Z} J_{1 / 2}(k a x)=2 \sqrt{\frac{2 x a}{\pi}} \frac{Z}{\left(x^{2} a^{2}+Z^{2}\right)^{2}} .
$$

The energy can again be expressed in closed form:

$$
U=-\frac{d^{2}}{8 Z^{3}}+\frac{d^{2}}{4 \pi Z^{3}}\left[\arctan \frac{a}{Z}+\frac{Z}{a} \frac{1+8 / 3(Z / a)^{2}-(Z / a)^{4}}{\left(1+Z^{2} / a^{2}\right)^{3}}\right] .
$$

This is always negative, but vanishes at infinity and at zero. Numerically, we find that the force changes sign at $Z=0.742358 a$. The reason why the energy vanishes when the dipole is centered in the aperture is clear: Then the electric field lines are perpendicular to the conducting sheet on the surface, and the sheet could be removed without changing the field configuration.

\section{Casimir-Polder Energy}

Our goal now is to find analytically the quantum (Casimir) analog of this classical repulsion. This was given a numerical study in Ref. 22. Here we want to offer an analytic counterpart. We will in this section be considering the Casimir-Polder (CP) interaction of an atom with a conducting body, which is in general given by

$$
U_{\mathrm{CP}}=-\int_{-\infty}^{\infty} d \zeta \operatorname{tr} \boldsymbol{\alpha} \cdot \boldsymbol{\Gamma}(\mathbf{r}, \mathbf{r} ; \zeta)
$$

where $\boldsymbol{\alpha}$ is the polarizability dyadic of the atom, located at position $\mathbf{r}$, and $\boldsymbol{\Gamma}$ is the Green's dyadic for the electromagnetic field at imaginary frequency $\zeta$ corresponding to the conducting body.

\subsection{Casimir-Polder force due to a conducting wedge}

Consider a polarizable atom located outside a conducting wedge, as shown in Fig. 6 . The interaction between a polarizable atom and a perfectly conducting half-plane is a special case of the vacuum interaction between such an atom and a conducting wedge. For an isotropic atom, the wedge was considered by Brevik, Lygren, and Marachevsky. ${ }^{26}$ In terms of the exterior dihedral angle of the wedge $\Omega$, with $p=\pi / \Omega$, 


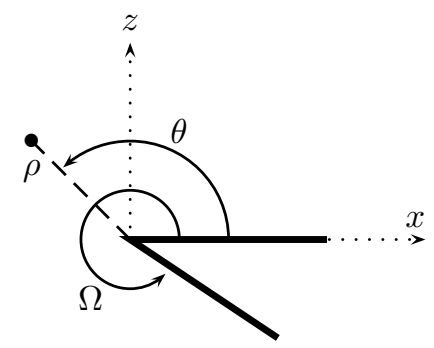

Figure 6. Polarizable atom, located at polar coordinates $\rho, \theta$, within a conducting wedge with dihedral angle $\Omega=\pi / p$.

the electromagnetic Green's dyadic has the form (here the translational direction is denoted by $y$, and one plane of the wedge lies in the $z=0$ plane, the other intersecting the $x z$ plane on the line $\theta=\Omega$ - see Fig. 6)

$$
\begin{aligned}
\boldsymbol{\Gamma}\left(\mathbf{r}, \mathbf{r}^{\prime}\right)= & 2 p \sum_{m=0}^{\infty}, \int \frac{d k}{2 \pi}\left[-\mathcal{M} \mathcal{M}^{\prime *}\left(\nabla_{\perp}^{2}-k^{2}\right)\right. \\
& \times \frac{1}{\omega^{2}} F_{m p}\left(\rho, \rho^{\prime}\right) \frac{\cos m p \theta \cos m p \theta^{\prime}}{\pi} e^{i k\left(y-y^{\prime}\right)} \\
& \left.+\mathcal{N N}^{\prime *} \frac{1}{\omega} G_{m p}\left(\rho, \rho^{\prime}\right) \frac{\sin m p \theta \sin m p \theta^{\prime}}{\pi} e^{i k\left(y-y^{\prime}\right)}\right] .
\end{aligned}
$$

The first term here refers to TE $(\mathrm{H})$ modes, the second to TM (E) modes. The prime on the summation sign means that the $m=0$ term is counted with half weight. In the polar coordinates in the $x z$ plane, $\rho$ and $\theta$, the $\mathrm{H}$ and $\mathrm{E}$ mode operators are

$$
\mathcal{M}=\hat{\boldsymbol{\rho}} \frac{\partial}{\rho \partial \theta}-\hat{\boldsymbol{\theta}} \frac{\partial}{\partial \rho}, \quad \mathcal{N}=i k\left(\hat{\boldsymbol{\rho}} \frac{\partial}{\partial \rho}+\hat{\boldsymbol{\theta}} \frac{\partial}{\rho \partial \theta}\right)-\hat{\mathbf{y}} \nabla_{\perp}^{2} .
$$

where the transverse Laplacian is

$$
\nabla_{\perp}^{2}=\frac{1}{\rho} \frac{\partial}{\partial \rho} \rho \frac{\partial}{\partial \rho}+\frac{1}{\rho^{2}} \frac{\partial^{2}}{\partial \theta^{2}} .
$$

In this situation, the boundaries are entirely in planes of constant $\theta$, so the radial Green's functions are equal to the free Green's function

$$
\frac{1}{\omega^{2}} F_{m p}\left(\rho, \rho^{\prime}\right)=\frac{1}{\omega} G_{m p}\left(\rho, \rho^{\prime}\right)=-\frac{i \pi}{2 \lambda^{2}} J_{m p}\left(\lambda \rho_{<}\right) H_{m p}^{(1)}\left(\lambda \rho_{>}\right),
$$

with $\lambda^{2}=\omega^{2}-k^{2}$. We will immediately make the Euclidean rotation, $\omega \rightarrow$ $i \zeta$, where $\lambda \rightarrow i \kappa, \kappa^{2}=\zeta^{2}+k^{2}$, so the free Green's functions become $-\kappa^{-2} I_{m p}\left(\kappa \rho_{<}\right) K_{m p}\left(\kappa \rho_{>}\right)$. 


\subsubsection{Completely anisotropic atom}

We start by considering the most favorable case for Casimir-Polder repulsion, where only $\alpha_{z z} \neq 0$. In the static limit, where the frequency dependence of the polarizability is neglected, then the only component of the Green's dyadic that contributes comes in as

$$
\begin{aligned}
\int \frac{d \zeta}{2 \pi} \Gamma_{z z}=\frac{2 p}{4 \pi^{3}} & \int d k d \zeta\left\{\left[\zeta^{2} \sin ^{2} \theta \sin ^{2} m p \theta-k^{2} \cos ^{2} \theta \cos ^{2} m p \theta\right]\right. \\
& \times \frac{m^{2} p^{2}}{\kappa^{2} \rho_{<} \rho_{>}} I_{m p}\left(\kappa \rho_{<}\right) K_{m p}\left(\kappa \rho_{>}\right) \\
& \left.-\left[k^{2} \sin ^{2} \theta \sin ^{2} m p \theta-\zeta^{2} \cos ^{2} \theta \cos ^{2} m p \theta\right] I_{m p}^{\prime}\left(\kappa \rho_{<}\right) K_{m p}^{\prime}\left(\kappa \rho_{>}\right)\right\} .
\end{aligned}
$$

Here we note that the off diagonal $\rho-\theta$ terms in $\boldsymbol{\Gamma}$ cancel. We have regulated the result by point-splitting in the radial coordinate. At the end of the calculation, the limit $\rho_{<} \rightarrow \rho_{>}=\rho$ is to be taken. Now the integral over the Bessel functions is given by

$$
\int_{0}^{\infty} d \kappa \kappa I_{\nu}\left(\kappa \rho_{<}\right) K_{\nu}\left(\kappa \rho_{>}\right)=\frac{\xi^{\nu}}{\rho_{>}^{2}\left(1-\xi^{2}\right)}
$$

where $\xi=\rho_{<} / \rho_{>}$. After that the $m$ sum is easily carried out by summing a geometrical series. Care must also be taken with the $m=0$ term in the cosine series. The result of a straightforward calculation leads to

$$
\int \frac{d \zeta}{2 \pi} \Gamma_{z z}=-\frac{\cos 2 \theta}{\pi^{2} \rho^{4}} \frac{1}{(\xi-1)^{4}}+\text { finite. }
$$

The divergent term is that of the vacuum without the wedge, so we must subtract this term off, leaving for the static Casimir-Polder energy

$$
U_{\mathrm{CP}}^{z z}=-\frac{\alpha_{z z}(0)}{8 \pi} \frac{1}{\rho^{4} \sin ^{4} p \theta}\left[p^{4}-\frac{2}{3} p^{2}\left(p^{2}-1\right) \sin ^{2} p \theta+\frac{\left(p^{2}-1\right)\left(p^{2}+11\right)}{45} \sin ^{4} p \theta \cos 2 \theta\right] \text {. }
$$

This result may also be easily derived from the closed form given by Lukosz. ${ }^{20}$

A small check of this result is that as $\theta \rightarrow 0$ (or $\theta \rightarrow \Omega$ ) we recover the expected Casimir-Polder result for an atom above an infinite plane:

$$
U_{\mathrm{CP}}^{z z} \rightarrow-\frac{\alpha_{z z}(0)}{8 \pi Z^{4}}
$$

in terms of the distance of the atom above the plane, $Z=\rho \theta$. This limit is also obtained when $p \rightarrow 1$, for when $\Omega=\pi$ we are describing a perfectly conducting infinite plane.

A very similar calculation gives the result for an isotropic atom, $\boldsymbol{\alpha}=\alpha \mathbf{1}$, which was first given by Brevik, Lygren, and Marachevsky: ${ }^{26}$

$$
U_{\mathrm{CP}}=-\frac{3 \alpha(0)}{8 \pi \rho^{4} \sin ^{4} p \theta}\left[p^{4}-\frac{2}{3} p^{2}\left(p^{2}-1\right) \sin ^{2} p \theta-\frac{1}{3} \frac{1}{45}\left(p^{2}-1\right)\left(p^{2}+11\right) \sin ^{4} p \theta\right] .
$$

Note that this is not three times $U_{\mathrm{CP}}^{z z}$ in above, because the $\cos 2 \theta$ factor in the last term in the latter is replaced by $-1 / 3$ here. 


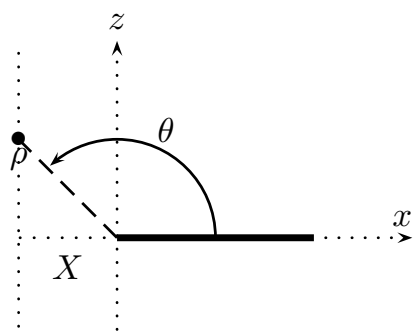

Figure 7. Polarizable atom, above a half conducting plane, free to move on a line perpendicular to the plane but a distance $X$ to the left of the plane.

\subsubsection{Repulsion by half-plane}

Let us consider the special case $p=1 / 2$, that is $\Omega=2 \pi$, the case of a semiinfinite conducting plane, illustrated in Fig. 7. This was the situation considered, for anisotropic atoms, in recent papers by Eberlein and Zietal. ${ }^{27,28}$ Consider a particle free to move along a line parallel to the $z$ axis, a distance $X$ to the left of the semi-infinite plane.

The half-plane $x<0$ constitutes an aperture of infinite width. With $X$ fixed, we can describe the trajectory by $u=X / \rho=-\cos \theta$, which variable ranges from zero to one. The polar angle is given by

$$
\sin ^{2} \frac{\theta}{2}=\frac{1+u}{2}
$$

The energy for an isotropic atom is given by

$$
U_{\mathrm{CP}}=-\frac{\alpha(0)}{32 \pi} \frac{1}{X^{4}} V(u)
$$

where

$$
V(u)=3 u^{4}\left[\frac{1}{(1+u)^{2}}+\frac{1}{u+1}+\frac{1}{4}\right] .
$$

The energy for the completely anisotropic atom is

$$
V_{z z}=\frac{1}{3} V(u)+\frac{u^{4}}{2}\left(1-3 u^{2}\right) .
$$

If we consider instead a cylindrically symmetric polarizable atom in which

$$
\boldsymbol{\alpha}=\alpha_{z z} \hat{\mathbf{z}} \hat{\mathbf{z}}+\gamma \alpha_{z z}(\hat{\mathbf{x}} \hat{\mathbf{x}}+\hat{\mathbf{y}} \hat{\mathbf{y}})=\alpha_{z z}(1-\gamma) \hat{\mathbf{z}} \hat{\mathbf{z}}+\gamma \alpha_{z z} \mathbf{1},
$$

where $\gamma$ is the ratio of the transverse polarizability to the longitudinal polarizability of the atom, the effective potential is

$$
(1-\gamma) V_{z z}+\gamma V
$$

and the $z$-component of the force on the atom is

$$
F_{z}^{\gamma}=-\frac{\alpha_{z z}(0)}{32 \pi} \frac{1}{X^{5}} u^{2} \sqrt{1-u^{2}} \frac{d}{d u}\left[\frac{1}{2} u^{4}(1-\gamma)\left(1-3 u^{2}\right)+\frac{1}{3}(1+2 \gamma) V(u)\right]
$$




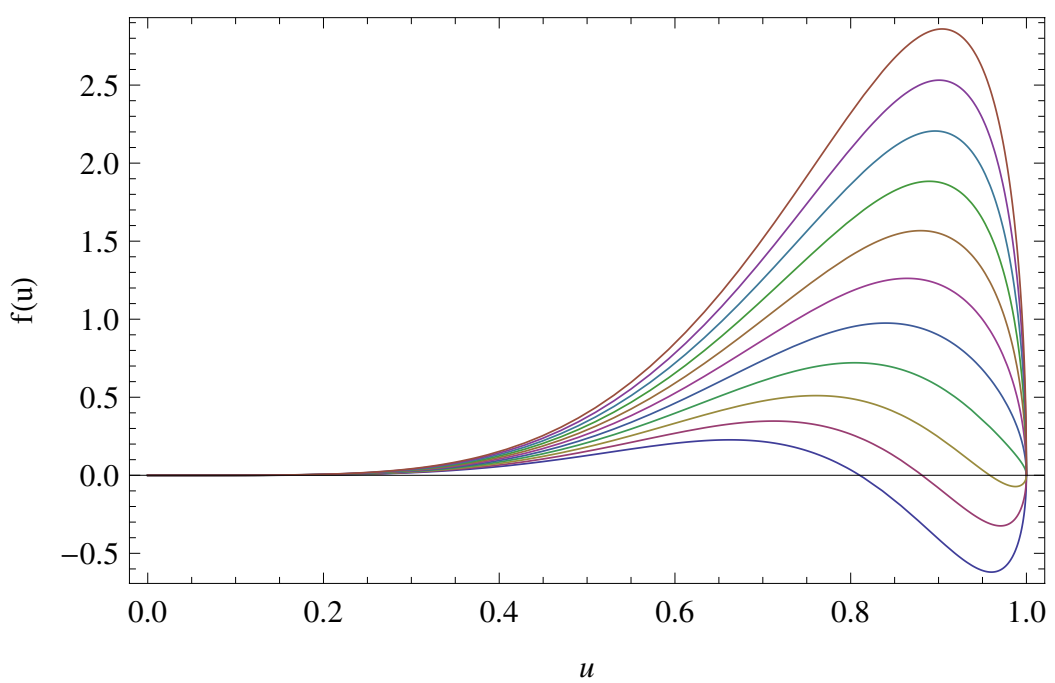

Figure 8. The $z$ component of the force between an anisotropic atom and a semi-infinite conducting plane. $F_{z}=-\alpha_{z z} /\left(32 \pi X^{5}\right) f(u)$ in terms of the variable $u=X / \rho=-\cos \theta$. Here the atom lies on the line $y=0, x=-X$, and $\rho$ is the distance from the edge of the plane and the atom. $f>0$ is attractive, $f<0$ repulsive. $\gamma$ goes from 0 to 1 by steps of 0.1 , from bottom to top. For $\gamma<1 / 4$ a repulsive regime always occurs when the atom is sufficiently close to the plane of the conductor.

where $V$ is given by (74). Note that the energy, or the quantity in square brackets in (78), only vanishes at $u=1$ (the plane of the conductor) when $\gamma=0$. Thus, the argument given in Levin et al. ${ }^{22}$ applies only for the completely anisotropic case. Figure 8 shows the dependence of $F_{z}$ on the polar angle. Figure 9 gives a finer resolution plot. The critical value of $\gamma_{c}=1 / 4$ marks the boundary between the regime where no repulsion occurs, and where repulsion occurs close to the plane of the conductor. It is interesting to observe that the same critical value of $\gamma$ occurs for the nonretarded (electrostatic) regime of a circular aperture, as follows from a simple computation based on the result of Eberlein and Zietal. ${ }^{27,28}$

$$
\begin{aligned}
U=- & \frac{1}{16 \pi^{2}} \int_{-\infty}^{\infty} d \zeta \alpha_{z z}(\zeta) \frac{1}{Z^{3}}\left\{(1+\gamma)\left(\frac{\pi}{2}+\arctan \frac{Z^{2}-a^{2}}{2 a Z}\right)\right. \\
& \left.+\frac{2 a Z}{\left(Z^{2}+a^{2}\right)^{3}}\left[(1+\gamma)\left(Z^{4}-a^{4}\right)-\frac{8}{3}(1-\gamma) a^{2} Z^{2}\right]\right\} .
\end{aligned}
$$

It is easy to see that this has a minimum for $z>0$, and hence there is a repulsive force close to the aperture, provided $\gamma<\gamma_{c}=1 / 4$.

\subsubsection{Repulsion by a wedge}

It is very easy to generalize the above result for a wedge, $p>1 / 2$. That is, we want to consider a strongly anisotropic atom, with only $\alpha_{z z}$ significant, to the left of a 


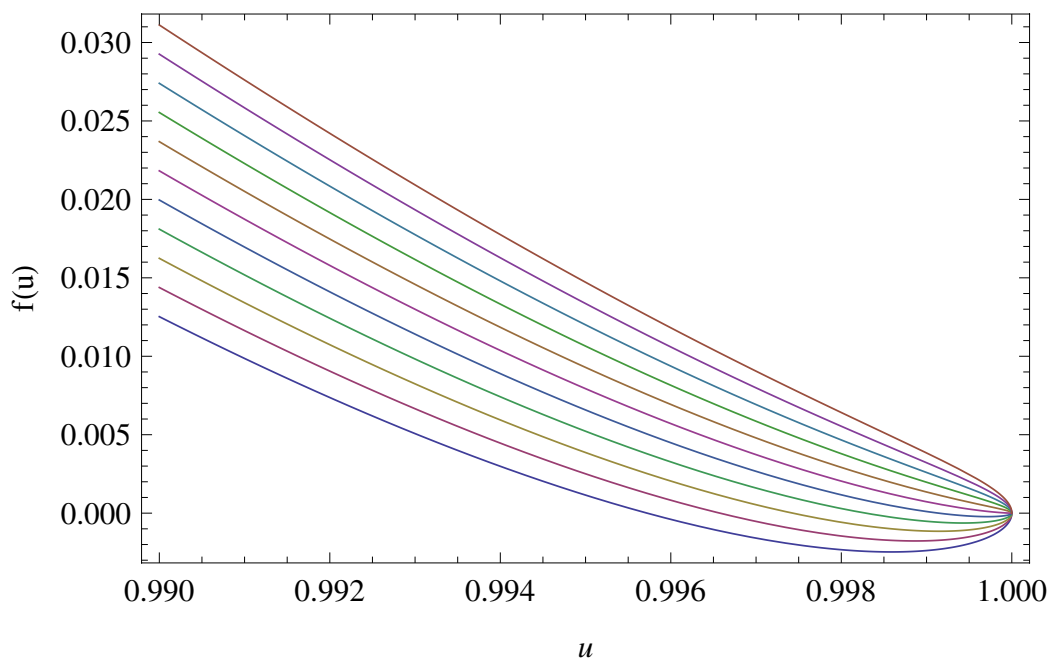

Figure 9. The region close to the plane, $1 \geq u \geq 0.99$, with $\gamma$ near the critical value of $1 / 4$. Here from bottom to top are shown the results for values of $\gamma$ from 0.245 to 0.255 by steps of 0.001 .

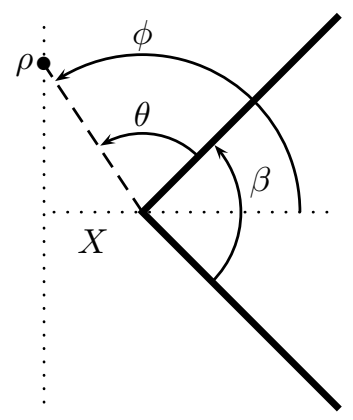

Figure 10. Polarizable atom outside a perfectly conducting wedge of opening angle $\beta$.

wedge of interior angle

$$
\beta=2 \pi-\Omega
$$

as shown in Fig. 10. We want the $z$ axis to be perpendicular to the symmetry axis of the wedge so the relation between the polar angle of the atom and the angle to the symmetry line is $\phi=\theta+\beta / 2$, where, as before, $\theta$ is the angle relative to the top surface of the wedge. The CP energy is changed only by the replacement $\cos 2 \theta$ by $\cos 2 \phi$, with no change in $\sin p \theta$. How does repulsion depend on the wedge angle $\beta$ ? Write for an atom on the line $x=-X$

$$
U_{\mathrm{CP}}^{z z}=-\frac{\alpha_{z z}(0)}{8 \pi X^{4}} V(\phi),
$$




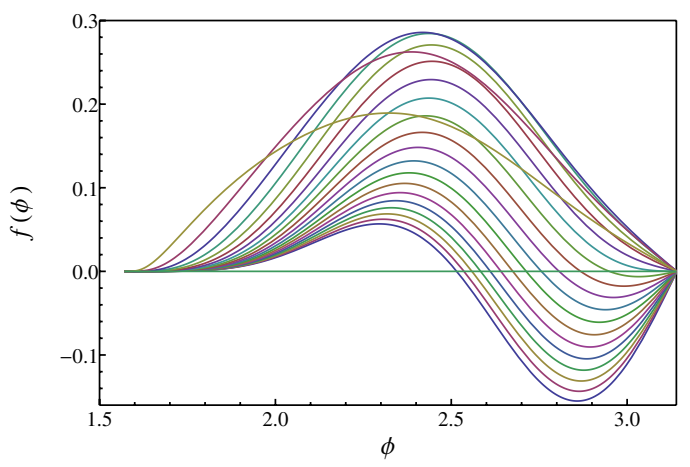

Figure 11. $\quad F_{z}$ for a completely anisotropic atom moving on a line perpendicular to the wedge. The different curves are for different values of the interior angle $\beta=n \pi / 20, n=0$ to 20 , from bottom up.

where

$$
V(\phi)=\cos ^{4} \phi\left[\frac{p^{4}}{\sin ^{4} \frac{\pi}{2} \frac{\phi-\beta / 2}{\pi-\beta / 2}}-\frac{2}{3} \frac{p^{2}\left(p^{2}-1\right)}{\sin ^{2} \frac{\pi}{2} \frac{\phi-\beta / 2}{\pi-\beta / 2}}+\frac{1}{45}\left(p^{2}-1\right)\left(p^{2}+11\right) \cos 2 \phi\right] .
$$

At the point of closest approach,

$$
V(\pi)=\frac{1}{45}\left(4 p^{2}-1\right)\left(4 p^{2}+11\right)
$$

so the potential vanishes at that point only for the half-plane case, $p=1 / 2$. The force in the $z$ direction is

$$
F_{z}=-\frac{\alpha_{z z}}{8 \pi} \frac{1}{X^{5}} \cos ^{2} \phi \frac{\partial V(\phi)}{\partial \phi} .
$$

Figure 11 shows the force as a function of $\phi$ for fixed $X$. It will be seen that the force has a repulsive region for angles close enough to the apex of the wedge, provided that the wedge angle is not too large. The critical wedge angle is actually rather large, $\beta_{c}=1.87795$, or about $108^{\circ}$. For larger angles, the $z$-component of the force exhibits only attraction.

\subsection{CP repulsion by cylinder not sphere}

Finally, we turn to the Casimir-Polder interaction between an anisotropic atom and an infinite cylinder, for the force on the atom along a line perpendicular to, and not intersecting, the cylinder, which is also polarizable only along the same direction. The situation is illustrated inf Fig. 12. The details will appear elsewhere. ${ }^{29}$ Figure 13 shows a plot of the CP energy, the upper curve being for the distance of closest approach to the cylinder axis $R$ being 5 times the cylinder radius $a$, the lower curve for the distance of closest approach 10 times the radius. Repulsion is clearly observed when $R / a=10$, but not for $R / a=5$. In contrast, for a conducting sphere, since 


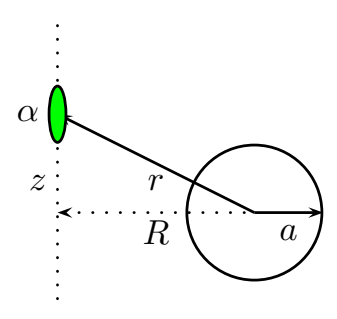

Figure 12. Interaction between an anisotropically polarizable atom and a conducting cylinder of radius $a$. The force on the atom along a line which does not intersect the cylinder is considered. If the atom is only polarizable in that direction, and the line lies sufficiently far from the cylinder, the force component along the line changes sign near the point of closest approach.

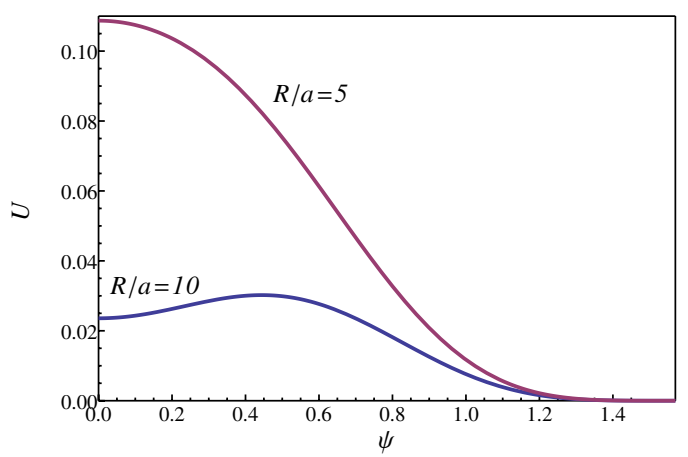

Figure 13. CP energy between a completely anisotropic atom and a cylinder. The motion of the atom, and its polarizability, are along a line perpendicular to the cylinder, and not intersecting with it. Here $\psi$ is the angle above the radial line perpendicular to the line of motion of the atom. That is, according to Fig. $12, \sin \psi=z / r$.

at large distances it looks like an isotropic polarizable atom (with both electric and magnetic polarizabilities), no repulsion on a completely anisotropic atom occurs.

\section{Conclusions}

- Casimir self-energies often exhibit repulsion, but general systematics are not yet completely worked out.

- Repulsion occurs between electric and magnetic conductors, or materials or metamaterials that mimic this behavior over a wide frequency range. This is extraordinarily difficult to achieve in practice.

- Intervening intermediate "density" materials can mimic repulsion.

- But true repulsion can be exhibited in Casimir-Polder situations with suitable anisotropies.

- New examples of Casimir and Casimir-Polder repulsion are still being discovered. 


\section{Acknowledgments}

We thank the US National Science Foundation, the US Department of Energy, and the European Science Foundation for partial support of this research.

\section{Bibliography}

1. J. Schwinger, Lett. Math. Phys. 1, 43 (1975).

2. B. A. Lippmann and J. Schwinger, Phys. Rev. 79, 469 (1950).

3. T. H. Boyer, Phys. Rev. A 9, 2078 (1974).

4. I. D. Dzyaloshinskii, E. M. Lifshitz, and L. P. Pitaevskii, Usp. Fiz. Nauk 73, 381 (1961) [English transl.: Soviet Phys. Usp. 4, 153 (1961)].

5. J. Munday, F. Capasso, and V. Persegian, Nature 457, 170 (2009).

6. T. H. Boyer, Phys. Rev. 174, 1764 (1968).

7. L. L. DeRaad, Jr. and K. A. Milton, Ann. Phys. (N.Y.) 136, 229 (1981).

8. C. M. Bender and K. A. Milton, Phys. Rev. D 50, 6547 (1994) [arXiv:hep-th/9406048].

9. P. Gosdzinsky and A. Romeo, Phys. Lett. B 441, 265 (1998) [arXiv:hep-th/9809199].

10. I. Brevik, V. N. Marachevsky and K. A. Milton, Phys. Rev. Lett. 82, 3948 (1999) [arXiv:hep-th/9810062].

11. I. Cavero-Peláez and K. A. Milton, Ann. Phys. (N.Y.) 320, 108 (2005) [arXiv:hepth/0412135].

12. I. Klich, Phys. Rev. D 61, 025004 (2000) [arXiv:hep-th/9908101].

13. K. A. Milton, A. V. Nesterenko, and V. V. Nesterenko, Phys. Rev. D 59, 105009 (1999).

14. K. A. Milton, Phys. Rev. D 68, 065020 (2003) [arXiv:hep-th/0210081].

15. I. Cavero-Peláez, K. A. Milton and K. Kirsten, J. Phys. A 40, 3607 (2007) [arXiv:hepth/0607154].

16. E. K. Abalo, K. A. Milton, and L. Kaplan, Phys. Rev. D 82, 125007 (2010) [arXiv:1008.4778].

17. J. Schwinger, L. L. DeRaad, Jr., K. A. Milton, and W.-y. Tsai, Classical Electrodynamics (Perseus, New York, 1998).

18. K. A. Milton and J. Schwinger, Electromagnetic Radiation (Springer, Berlin, 2006).

19. E. Elizalde, S. D. Odintsov, A. Romeo, A. A. Bytsenko, and S. Zerbini, Zeta Regularization Techniques with Applications (World Scientific, Singapore, 1994).

20. W. Lukosz, Z. Phys. 262, 327 (1971).

21. J. Ambjørn and S. Wolfram, Ann. Phys. (N.Y.) 147, 1 (1983).

22. M. Levin, A. P. McCauley, A. W. Rodriguez, M. T. Homer Reid, and S. G. Johnson, Phys. Rev. Lett. 105, 090403 (2010) [arXiv:1003.3487].

23. K. A. Milton, E. K. Abalo, P. Parashar, N. Pourtolami, I. Brevik, and S. Å Ellingsen, Phys. Rev. A 83, 062507 (2011) [arXiv:1103.4386].

24. A. D. Khanzhov, Inzherero-Fizicheskia Zhurnal 11, 658 (1966) [Engl. Transl.: J. Eng. Phys. Thermophys. 11, 370 (1966)].

25. E. C. Titchmarsh, Theory of Fourier Integrals (Oxford, 1948).

26. I. Brevik, M. Lygren, and V, N. Marachevsky, Ann. Phys. (N.Y.) 267, 134 (1998) .

27. C. Eberlein and R. Zietal, Phys. Rev. A 80, 012504 (2009) [arXiv:0904.4785].

28. C. Eberlein and R. Zietal, Phys. Rev. A 83, 052514 (2011) [arXiv:1103.2381

29. K. A. Milton, P. Parashar, N. Pourtolami, and I. Brevik, arXiv:1111.4224. 